\title{
Persulfate-enhanced Photocatalytic Degradation of Methylene Blue
}

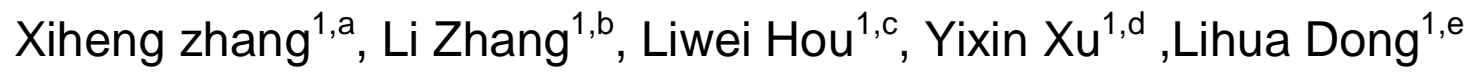 \\ ${ }^{1}$ No. 1550 HaiGang Avenue, Shanghai Maritime Universitiy, College of Ocean Science and \\ Engineering, Shanghai 201306, China \\ axhengzhang@163.com, bhangli@shmtu.edu.cn, ${ }^{\mathrm{c}}$ liweihouwhu@163.com \\ dxuyixin@shmtu.edu.cn, ${ }^{\mathrm{e}}$ Ihdong@shmtu.edu.cn
}

Key words: MB, sodium persulfate, degradation;

Abstract. Based on the new advanced oxidation technology activated by sulfate radicals, this work established a promising method on degradation of Methylene Blue (MB). Different effects of operating parameters such as the threshold concentration of sodium persulfate(SPS), initial $\mathrm{pH}$ value, coexisting ions and ultraviolet(UV) intensity, were studied in aim to obtain an optimal condition of MB degradation. Results showed that the increase of SPS concentration and UV intensity, as well as the decrease of initial $\mathrm{pH}$ value could significantly enhance MB degradation rate, but coexisting ions did greatly inhibit efficiency to an extent which was closely dependent upon the ion content. When the concentration of SPS was $0.5 \mathrm{mM}$ and the initial $\mathrm{pH}$ value is 3 under the irradiation of a 5W UV light, the degradation rate of MB reached the maximum value of $95 \%$ after an hour. Furthermore, Total Organic Carbon (TOC) of the MB system decreased from the initial $92.4 \mathrm{mg} / \mathrm{L}$ to $7.24 \mathrm{mg} / \mathrm{L}$.

\section{Introduction}

Gradually accumulated in the environment, dye wastewater, as a typical industrial pollutants, is regarded as hazardous polluted source, which will pose great environmental threats to the aquatic ecosystem as a result of severely carcinogenic, and mutagenic effects ${ }^{[1,2]}$. Since the pollutants are heterocyclic compounds with high non-biodegradable organic concentration and great stability. It is difficult to effectively degrade and control it. Consequently, processing methods and treatments of dye wastewater has gained significant concern in the field of environmental protection.

For decades, there exist an increasing number of disadvantages in the traditional processing methods. To eliminate the drawbacks, Advanced Oxidation Processes (AOPs) have been put into practice as potential remediation to address organic pollution in soil and water. This method is based on radical mechanism; hydroxyl $\operatorname{radical}(\cdot \mathrm{OH})$ and sulfate $\operatorname{radical}\left(\mathrm{SO}_{4}{ }^{-}\right)$, are two typical radical oxidants, which are kinetically slow in ordinary conditions and often induced by photolysis $^{[3]}$, microwaves ${ }^{[4]}$, heat ${ }^{[5,6]}$, electron beam ${ }^{[7,8]}$, ultrasonication ${ }^{[9]}$, alkaline ${ }^{[10,11]}$ etc. Via chemical reaction with those emerging oxidant, organic pollutants in wastewater difficult to keep away are converted to non-toxic and small molecules, or directly mineralized into harmless inorganic molecules such as $\mathrm{CO}_{2}, \mathrm{H}_{2} \mathrm{O}$.

$\mathrm{SO}_{4} \bullet^{-}$compared to $\cdot \mathrm{OH}$, is not only major oxidizing specie with higher standard redox potential $\left(\mathrm{E}_{\mathrm{SO} 4 \cdot-/ \mathrm{SO} 42_{-}^{-}}=2.5-3.1 \mathrm{~V}, \mathrm{E} \cdot \mathrm{OH} / \mathrm{OH}=2.7 \mathrm{~V}\right)$, but has greater property, whose degradation effect is more significant and can be used in wider range of $\mathrm{pH}$ value(5-8). So persulfate-activated AOPs have become more popular in the field of advanced oxidation

Considering the solubility, stability and other factors, SPS and typical aromatic MB as dye wastewater pollutants were determined (The properties are shown in Table 1). Different operating 
conditions such as initial concentration of SPS, initial $\mathrm{pH}$ value, UV intensity and the concentration of anion influence on the degradation rate of $\mathrm{MB}$, to find optimum conditions. Finally, via the change of TOC, an optimal degradation is determined.

\begin{tabular}{ccccc}
\hline Name & Formula & Molecule Weight & Solubility in water & Melting point \\
\hline MB & $\mathrm{C}_{16} \mathrm{H}_{18} \mathrm{ClN}_{3} \mathrm{~S}$ & 319.85 & $\geq 10 \mathrm{~g} / 100 \mathrm{ml}$ & $190^{\circ} \mathrm{C}$ \\
\hline
\end{tabular}

Table 1 : Important Properties of MB

\section{Method and Analysis}

Reactor

Experiments were performed in a 2-L glass photo-reactor involving four sample exits. The light source was a UV light $(5 \mathrm{~W}, 10 \mathrm{~W}$ and $40 \mathrm{~W})$, which was immersed partly in MB solution. A micro-air compressor bubbled air through a distributor from the reactor bottom into the solution for well mixing. Samples were taken out by both sides of the rector (shown in Fig.1).

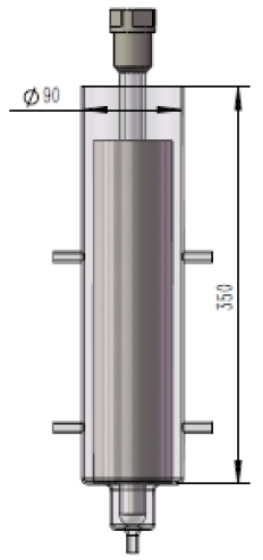

Fig. 1 Photo-reacter

\section{Reagents}

Analytical-Reagent-grade chemicals and ultrapure water produced from a Milli-Q biocel system $(18.3 \mathrm{M} \cdot \mathrm{cm})$ were used to prepare chemical reagents including MB sodium hydrate, sulfuric $\operatorname{acid}\left(\rho=18.4 \mathrm{~g} / \mathrm{cm}^{3}\right)$, sodium sulfate, sodium chloride, sodium nitrate, sodium persulfate. They are all purchased from Sinopharm Chemical Company.

Procedure

First, a $600 \mathrm{~mL}$ solution containing initial concentration of $10 \mathrm{mg} / \mathrm{L} \mathrm{MB}$ within the range of typical concentration in wastewaters was prepared. If necessary The $\mathrm{pH}$ was adjusted to the desired value $(3,5,7,9,11)$ by means of a $\mathrm{pH}$ meter (Mettler ToleDo) using diluted $3 \mathrm{~mol} / \mathrm{L} \mathrm{H}_{2} \mathrm{SO}_{4}$ or $\mathrm{NaOH}$ solutions. After being transferred into the reactor, appropriate amount of SPS $(0.1,0.2,0.5,1,2,5 \mathrm{mM})$ were added and the light was switched on to initiate irradiation .The total reaction lasted for $1 \mathrm{~h}$ and many samples were taken to analyze at certain time $(0,1,2,4,6,8,10,15,20,25,30,40,50,60 \mathrm{~min})$. When reaction came to an end, the remained solution was transferred to a $500 \mathrm{~mL}$ beaker for further analysis(TOC). 
Analytical methods

Degradation rate of $\mathrm{MB}$ was analyzed by a UV-Vis spectrophotometer (DR/2100). The wavelength of MB was $665.18 \mathrm{~nm}$. According to Beer-Lambert Law, Absorbance is proportional to the concentration. Consequently, degradation $\operatorname{rate}\left(\mathrm{C}_{\mathrm{t}} / \mathrm{C}_{0}\right)$ is calculated by the following formula : $\mathrm{A}=\mathrm{C}_{\mathrm{t}} / \mathrm{C}_{0}\left(\mathrm{C}_{0}\right.$ for the degradation $\mathrm{MB}$ solution absorbance; $\mathrm{C}_{\mathrm{t}}$ for photodegradation solution time $\mathrm{t}$ after $\mathrm{MB}$ solution absorbance.)

TOC was measured by a TOC analyzer (Multi N/C 3100, Analytik Jena, Germany).

\section{Results and Discussion}

Effect of initial concentration of SPS

The effect of SPS on the degradation rate of MB is shown in Fig.2. the initial concentration of SPS was determined in the following concentration : $0.0,0.1,0.5,1.0,5.0 \mathrm{mM}$.It is evident that the degradation rates increases greatly from $40 \%$ to $95 \%$ with a corresponding increase in concentration of SPS from 0mM to $5 \mathrm{mM}$, and the highest degradation rate is achieved at $90 \%$ when SPS is $5 \mathrm{mM}$. However, adding too much SPS can only slightly improve the degradation (only 10\%), still causing large waste of SPS. Due to the cost and profits, the concentration of SPS is determined at $0.5 \mathrm{mM}$.

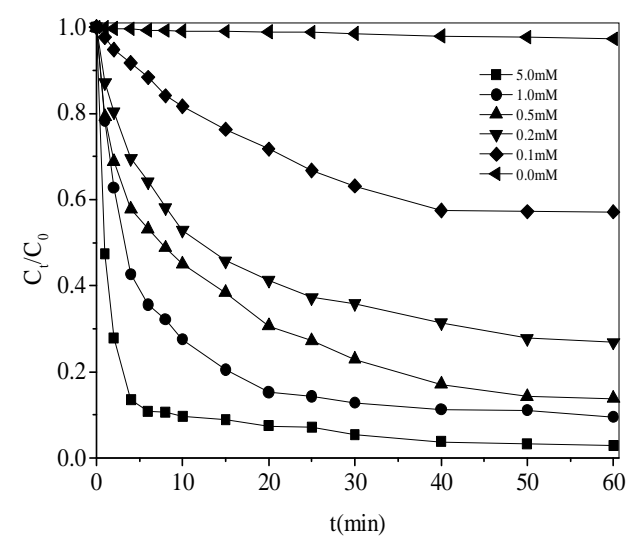

Fig.2. The effect of SPS on the MB degradation when $\mathrm{MB}$ conc. $=10 \mathrm{mg} / \mathrm{L}$; SPS conc $=0.5 \mathrm{mM} ; \mathrm{pH}=3 ; \mathrm{UVlight}=5 \mathrm{~W}$. Initial SPS conc. = $0.0,0.1,0.2,0.5,1.0 \mathrm{mM}$.

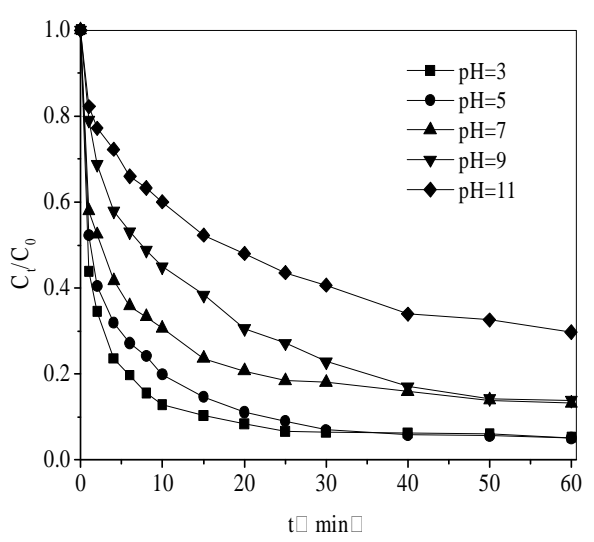

Fig.3. The effect of $\mathrm{pH}$ on the MB degradation rate when $\mathrm{MB}$ conc.$=10 \mathrm{mg} / \mathrm{L} ;$ SPS conc. $=0.5 \mathrm{mM}$; UV light $=5 \mathrm{~W}$. $\mathrm{pH}$ value was adjusted to the $3,5,7,9,11$.

Effect or IIIItial pп

The initial $\mathrm{pH}$ has a significant influence on $\mathrm{MB}$ degradation rate. With the increasing of initial $\mathrm{pH}$ value of $\mathrm{MB}$ solution from 3 to 11 , the $\mathrm{C}_{\mathrm{t}} / \mathrm{C}_{0}$ decreases accordingly. In Fig.3, it is demonstrated that when $\mathrm{pH}$ value is adjusted to 3 , the degradation rate of $\mathrm{MB}$ reaches maximum $(0.9479)$, much higher than that in the neutral and alkaline conditions (comparatively $0.868,0.702$ ).

In neutral or alkaline conditions, $\mathrm{SO}_{4}{ }^{-}$species may undergo reactions with $\mathrm{H}_{2} \mathrm{O}$ or $\mathrm{OH}^{-}$to generate $\mathrm{OH} \bullet$ according to Eq. 1 and Eq.2, respectively. With $\mathrm{SO}_{4}{ }^{-}$converting to $\mathrm{SO}_{4}{ }^{2-}, \mathrm{OH} \bullet$ is produced. Since $\mathrm{OH} \bullet$ has lower redox potential than that of $\mathrm{SO}_{4}{ }^{-}$, the presence of $\mathrm{OH} \bullet$ and $\mathrm{SO}_{4}{ }^{2-}$ can result inhibition of the reactivity of $\mathrm{SO}_{4}{ }^{-}$compounds ${ }^{[11]}$. The whole photocatalytic reaction system is dominated by heterogeneous catalysis induced by $\mathrm{OH} \bullet$, therefore, a considerable reduction is observed accordingly. 


$$
\begin{array}{ll}
\mathrm{SO}_{4}{ }^{-}+\mathrm{H}_{2} \mathrm{O} \rightarrow \mathrm{H}^{+}+\mathrm{SO}_{4}{ }^{2-}+\bullet \mathrm{OH} & \text { Eq.1 } \\
\mathrm{SO}_{4} \bullet^{-}+\mathrm{OH}^{-} \rightarrow \mathrm{SO}_{4}{ }^{2-}+\bullet \mathrm{OH} & \text { Eq.2 }
\end{array}
$$

Meanwhile, when $\mathrm{pH}<7$, homogeneous catalysis is dominated with $\mathrm{SO}_{4} \bullet^{-}$, which is confirmed that the acidic media promotes the higher $\mathrm{SO}_{4}{ }^{-}$generation(Eq.3)

$$
\begin{array}{ll}
\mathrm{S}_{2} \mathrm{O}_{8}{ }^{2-}+\mathrm{H}^{+} \rightarrow \mathrm{SO}_{4}{ }^{--}+\mathrm{HSO}_{4}{ }^{-} \quad \text { Eq.3 }
\end{array}
$$

With the increase of released $\mathrm{H}^{+}$, the degradation rate has become $8 \%$ higher. Although the degradation rate at $\mathrm{pH} 5(93 \%)$ almost equals to that at $\mathrm{pH} 3(94 \%)$, the velocity of $\mathrm{MB}$ degradation is relatively slow at 5 . So considering the highest efficiency of $\mathrm{MB}$, optimum initial $\mathrm{pH}$ value can be obtained at 3 .

\section{Effect of UV intensity}

As is shown in Fig.4, UV intensity also has impact on degradation rate. When UV lights power changes from $5 \mathrm{~W}$ to $40 \mathrm{~W}$, a mild increase occurs. Degradation rate changes gradually but not obviously from 0.948 to 0.994 , only increased $4.4 \%$. This positive correlation to the power of UV light can be explained that more UV photons will be stimulated with higher UV intensity, thus promoting more sulfate radicals. Taking the energy consumption and cost into account, the appropriate power of UV light is determined to $5 \mathrm{~W}$.

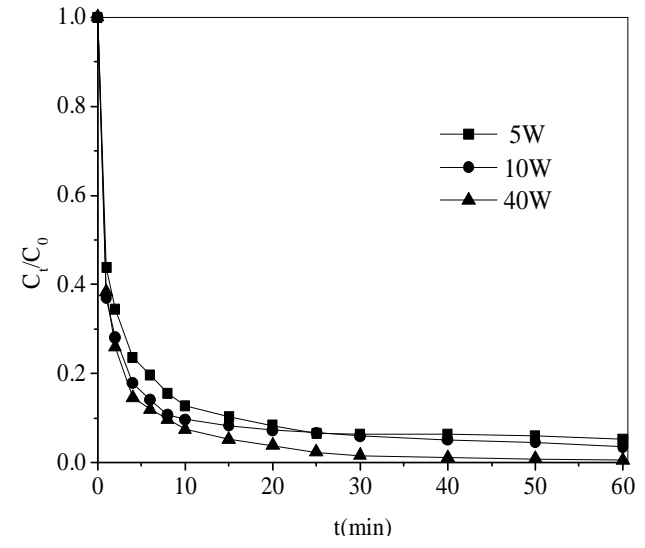

Fig.4. The Effect of UV light power on the MB degradation when $\mathrm{MB}$ conc. $=10 \mathrm{mg} / \mathrm{L}$; SPS conc. $=0.5 \mathrm{mM} ; \mathrm{pH}=3$; UV light was chosen respectively $5 \mathrm{~W}, 10 \mathrm{~W}$ and $40 \mathrm{~W}$.

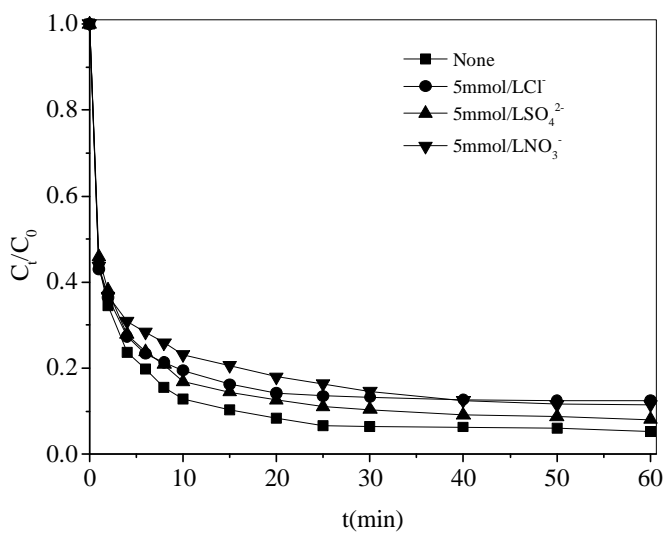

Fig.5. The effect of ions in the solution on

MB degradation when $\mathrm{MB}$ conc.$=10 \mathrm{mg} / \mathrm{L} ;$ SPS conc.$=0.5 \mathrm{mM}$; $\mathrm{pH}=3$; UV light $=5 \mathrm{~W}$.

Effect of ions in the solution

Experiments with different common ions in the solution show the importance of this parameter (Fig.4.-Fig.5.), the inhibiting effect of different anions on the degradation rate of MB is reported in the order as follow: nitrate $(0.875)<$ sulfate $(0.885)<$ chloride ions $(0.929)$. Among these, the chloride ions are obviously the most abundant species in the SPS oxidation system. It is pointed out that the similar inhibitory effect of three ions is caused by the competition between the sulfate radicals and the target pollutants $\mathrm{MB}$. Taking chloride ions as an example, $\mathrm{SO}_{4}{ }^{-}$can react with $\mathrm{Cl}^{-}$ to form the $\mathrm{Cl} \bullet$, which will subsequently transform into $\mathrm{Cl}_{2}{ }^{-}$, as shown in Eq.4 and Eq.6 ${ }^{[12,13,14]}$. Moreover, a plausible explanation can also be derived from the inhibition of $\mathrm{NO}_{3}{ }^{-}$and $\mathrm{SO}_{4}{ }^{2-}$ (Eq. 5) 


$$
\begin{array}{lr}
\mathrm{Cl}^{-}+\mathrm{SO}_{4} \bullet-\mathrm{Cl}^{-}+\mathrm{SO}_{4}{ }^{2-} \quad \mathrm{k}=4.7 \times 108 \mathrm{M}^{-1} \mathrm{~S}^{-1} & \text { Eq.4 } \\
\mathrm{NO}_{3}{ }^{-}+\mathrm{SO}_{4}{ }^{-} \rightarrow \mathrm{NO}_{3} \cdot+\mathrm{SO}_{4}{ }^{2-} & \text { Eq. } 5 \\
\mathrm{Cl} \bullet+\mathrm{Cl}^{-} \rightarrow \mathrm{Cl}_{2}{ }^{-} & \mathrm{k}=8 \times 10^{9} \mathrm{M}^{-1} \mathrm{~s}^{-1}
\end{array}
$$

In order to ascertain the inhibiting effect of anions, further photocatalytic experiments were conducted on different concentrations of chloride ion $(0.5,5,50,200 \mathrm{mM})$. The result shown in Fig.6. suggests that the higher concentration of $\mathrm{Cl}^{-}$leads to greater inhibition in the degradation of $\mathrm{MB}$ from $96 \%$ to $80 \%$,that is to say, causing more quenching of SO4 ${ }^{-}$.

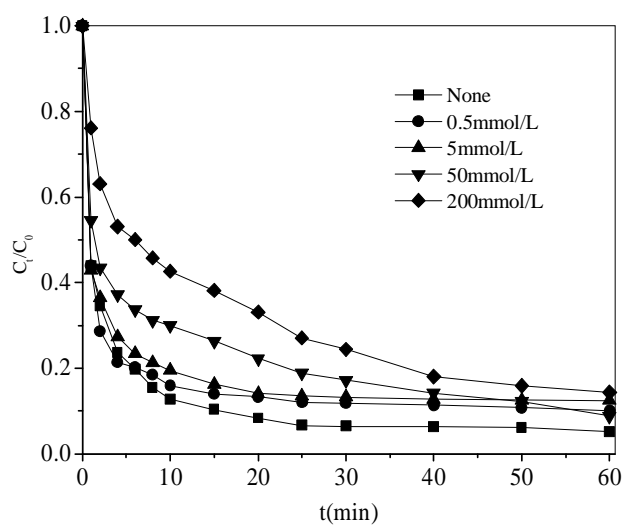

Fig.6. The effect of $\mathrm{Cl}^{-}$concentration on $\mathrm{MB}$ degradation when $\mathrm{MB}$ conc. $=$ $10 \mathrm{mg} / \mathrm{L} ;$ SPS conc. $=0.5 \mathrm{mM} ; \mathrm{pH}=3$; UV light $=5 \mathrm{~W}$. The chloride ion conc. $=0.5,5,50,200 \mathrm{mM}$.

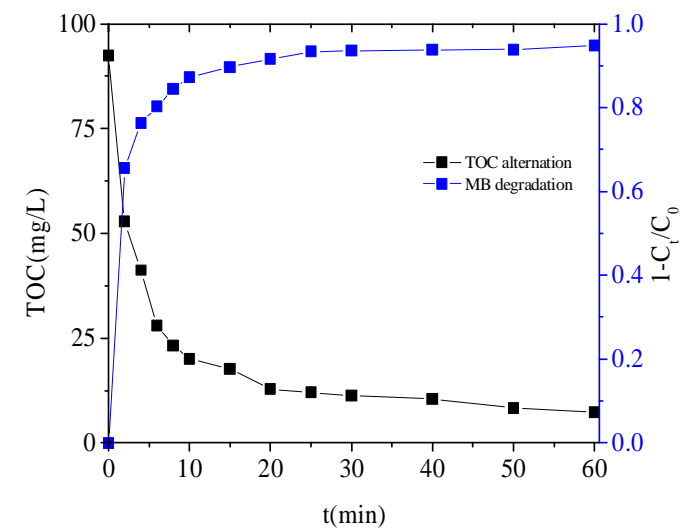

Fig.7. TOC alternation and degradation rate of $\mathrm{MB}$ when $\mathrm{MB}$ conc.$=10 \mathrm{mg} / \mathrm{L}$; SPS conc.$=$ $0.5 \mathrm{mM} ; \mathrm{pH}=3$; UV light=5W.

MB mineralization

The result reveals that initial TOC is $92.4 \mathrm{mg} / \mathrm{L}$. In the treatment, a fast primary mineralization stage is observed in the first $40 \mathrm{~min}$ of the reaction, where the organic matter in the carbohydrates is almost degraded. TOC removal was $88.4 \%$. After an hour, TOC is $7.24 \mathrm{mg} / \mathrm{L}$ and the mineralization reaches to the final value of $92.1 \%$ (Shown in Table 2 and Fig.7). At that moment, MB solution is almost degraded. In a word, SPS-induced advanced oxidation is more efficient than other photocatalytic system, such as $\mathrm{UV}-\mathrm{TiO}_{2}$. The degradation rate increases up to $5 \%$ in the same conditions. $^{[15]}$

\section{Conclusions}

This work demonstrates that it is feasible to degrade MB by emerging sodium persulfate activated advanced oxidation process. Different operating conditions were investigated and results reveal that the decrease of initial $\mathrm{pH}$ value and the increase of SPS addition or UV intensity favor the enhancement of the degradation rate. The anions (chloride, sulfate and nitrate) serving as radical scavengers in the solution, to some extent, will certainly inhibit the degradation rate of MB. The order of inhibition is nitrate < sulfate < chloride. With the increase of the amount of chlorine ion, inhibiting effect becomes greater. The degradation rate reaches maximum $95 \%$ and $\mathrm{MB}$ mineralization is up to $92.1 \%$ under the irradiation of a $5 \mathrm{~W}$ of UV light when the concentration of 
SPS is $0.5 \mathrm{mM}$ and the initial $\mathrm{pH}$ value is 3 . This optimal method will be urgently taken prior to the application to degrade similar aromatic pollutants.

\section{Acknowledgements}

This work was financially supported by the Shanghai Science and Technology Innovation Action Plan (No. 14DZ1205302) and The Project Sponsored by the Scientific Research Foundation for the Returned Overseas Chinese Scholars, State Education Ministry (No.20150285).

\section{References}

[1]X. Miao, F. Bishay, M. Chen, C.D. Metcalfe, Occurrence of antimicrobials in the final effluents of wastewater treatment plants in Canada, Environ. Sci. Technol.38 (2004) 3533-3541.

[2]P.Wang, Y.L. He, C.H. Huang, Oxidation of fluoroquinolones antibiotics structurally related amines by chlorine dioxide: reaction kinetics product and pathway evaluation, Water Res. 44 (2010) 5989-5998.

[3]Y.T. Lin, C.J. Liang, J.H. Chen, Feasibility study of ultraviolet activated persulfateoxidation of phenol, Chemosphere 82 (2011) 1168-1172.

[4] S.Y. Yang, P. Wang, X. Yang, G. Wei, W.Y. Zhang, L. Shan, A novel advanced oxidation process to degrade organic pollutants in wastewater: microwave activated persulfate oxidation, J. Environ. Sci.-China 21 (2009) 1175-1180.

[5]K.C. Huang, R.A. Couttenye, G.E. Hoag, Kinetics of heat-assisted persulfate oxidation of methyl tert-butyl ether (MTBE), Chemosphere 49 (2002) 413-420.

[6]R.H. Waldemer, P.G. Tratnyek, R.L. Johnson, J.T. Nurmi, Oxidation of chlorinated ethenes by heat-activated persulfate: kinetics and products,

Environ. Sci. Technol. 41 (2007) 1010-1015.

[7]J.Criquet, N.K.V. Leitner, Electron beam irradiation of aqueous solution ofpersulfate ions, Chem. Eng. J. 169 (2011) 258-262.

[8]S.O.B. Boukari, F. Pellizzari, N.K.V. Leitner, Influence of persulfate ions on the removal of phenol in aqueous solution using electron beam irradiation, J.Hazard. Mater. 185 (2011) 844-851.

[9] P. Gayathri, R.P.J. Dorathi, K. Palanivelu, Sonochemical degradation of textile dyes in aqueous solution using sulphate radicals activated by immobilized cobalt ions, Ultrason. Sonochem. 17 (2010) 566-571.

[10]O.S. Furman, A.L. Teel, R.J. Watts, Mechanism of base activation of persulfate, Environ. Sci. Technol. 44 (2010) 6423-6428.

[11]O.S. Furman, A.L. Teel, M. Ahmad, M.C. Merker, R.J. Watts, Effect of basicity onpersulfate reactivity, J. Environ. Eng. ASCE 137 (2011) 241-247.

[12]G.V. Buxton, C.L. Greenstock, W.P. Helman, A.B. Ross, Critical review of rate constants for reactions of hydrated electrons, hydrogen atoms and hydroxyl radicals $(\cdot \mathrm{OH} / \bullet \mathrm{O})$ in aqueous solution, J. Phys. Chem. Ref. Data 17 (1988) 513-531.

[13]V. Nagarajan, R.W. Fessenden, Flash photolysis of transient radicals. 1. X2-withX = Cl, Br, I, and SCN, J. Phys. Chem. 89 (1985) 2330-2335.

[14]X.Yu, Critical evaluation of rate constants and equilibrium constants of hydro-gen peroxide photolysis in acidic aqueous solutions containing chloride ions, J.Phys. Chem. Ref. Data 33 (2004) $747-76$ 
[15]ZHOU Hua-feng;LIANG Dian-wei;SUN Yi-rui;Shenyang University of Chemical Technology; Photocatalytic Degradation of Methylene Blue Over TiO2, Liaoning Chemical Industry, 2016.03.017 